OPEN ACCESS

Edited by:

Satoshi Hirata

Kyoto University, Japan

Reviewed by:

Yuji Ikegaya,

The University of Tokyo, Japan

Fuat Balcl,

Koç University, Turkey

*Correspondence:

Sorinel A. Oprisan oprisans@cofc.edu

Specialty section:

This article was submitted to

Perception Science,

a section of the journal

Frontiers in Neuroscience

Received: 24 March 2018

Accepted: 11 July 2018

Published: 07 August 2018

Citation:

Oprisan SA, Buhusi M and Buhusi CV

(2018) A Population-Based Model of the Temporal Memory in the

Hippocampus.

Front. Neurosci. 12:521. doi: 10.3389/fnins.2018.00521

\section{A Population-Based Model of the Temporal Memory in the Hippocampus}

\author{
Sorinel A. Oprisan ${ }^{1 *}$, Mona Buhusi ${ }^{2}$ and Catalin V. Buhusi ${ }^{2}$ \\ ${ }^{1}$ Department of Physics and Astronomy, College of Charleston, Charleston, SC, United States, ${ }^{2}$ Interdisciplinary Program in \\ Neuroscience, Department of Psychology, Utah State University, Logan, UT, United States
}

Spatial and temporal dimensions are fundamental for orientation, adaptation, and survival of organisms. Hippocampus has been identified as the main neuroanatomical structure involved both in space and time perception and their internal representation. Dorsal hippocampus lesions showed a leftward shift (toward shorter durations) in peak-interval procedures, whereas ventral lesions shifted the peak time toward longer durations. We previously explained hippocampus lesion experimental findings by assuming a topological map model of the hippocampus with shorter durations memorized ventrally and longer durations more dorsal. Here we suggested a possible connection between the abstract topological maps model of the hippocampus that stored reinforcement times in a spatially ordered memory register and the "time cells" of the hippocampus. In this new model, the time cells provide a uniformly distributed time basis that covers the entire to-be-learned temporal duration. We hypothesized that the topological map of the hippocampus stores the weights that reflect the contribution of each time cell to the average temporal field that determines the behavioral response. The temporal distance between the to-be-learned criterion time and the time of the peak activity of each time cell provides the error signal that determines the corresponding weight correction. Long-term potentiation/depression could enhance/weaken the weights associated to the time cells that peak closer/farther to the criterion time. A coincidence detector mechanism, possibly under the control of the dopaminergic system, could be involved in our suggested error minimization and learning algorithm.

Keywords: hippocampus, topological map, scale invariance, time cells, computer simulations, neural networks

\section{INTRODUCTION}

Spatial and temporal dimensions are fundamental for orientation, adaptation, and survival of organisms. Hippocampus has been identified as the main neuroanatomical structure involved both in space and time perception and their internal representation. It has been hypothesized that hippocampus may be in fact involved in conceptual understanding of many other dimensions (Schapiro et al., 2015; Schiller et al., 2015). While hippocampus encodes and processes information regarding spatial location and temporal durations, the same or similar computational structures could be used for encoding and processing the "state" of the brain associated to more abstract tasks 
than spatial location, such as extracting patterns from apparently random events (Garvert et al., 2017). It has been suggested that the same or a similar computational algorithm that establishes a metric space for the abstract dimension of time could be employed to create metric spaces and ordered categories for cognitive maps that relate other abstract concepts (Howard et al., 2014).

Temporal Dimension and Time Cells. One of the most used experimental paradigms when investigating temporal perception is the treadmill running, which allows precise correlation of neural firing with the spatial location and temporal duration. In vivo recordings from hippocampus and entorhinal cortex showed that neurons ramp-up their firing only at specific temporal locations during a behavioral tasks (Pastalkova et al., 2008; MacDonald et al., 2011; Kraus et al., 2013; Wang et al., 2015). This activity is similar to the hippocampus "place cells" that ramp-up their firing only when the subject is in a specific spatial location (O'Keefe, 1976; O'Keefe and Recce, 1993; Mathis et al., 2012). This is the reason the hippocampus cells that fire at a specific time during a behavioral test are called "time cells." Single-cell recordings from hippocampus suggested a clear correlation of the firing rate of the time cells with the to-be-timed duration (MacDonald et al., 2011). They found that different time cells selectively and repeatably peak at specific moments during the to-be-timed duration (MacDonald et al., 2011). In similar experiments involving a reward after a specific delay time, it has been found also that neurons in the rodent hippocampus selectively fired at specific times after the beginning of a delay period (Pastalkova et al., 2008). It has been shown that some time cells are involved in timing absolute durations whereas others fire relative to a specific temporal marker (MacDonald et al., 2011). Neurons that fired during the to-be-timed interval were typically striking in their selectivity to specific moments in the time interval. These time cells fire at successive moments within a temporally defined period. A hallmark of the time cells experiments is that the spread of the firing interval, i.e., the width of the Gaussianlike activity, for each time cell is proportional to the time of the peak activity. Additional experimental measurements also confirmed the proportional spread in time fields of time cells for longer durations (Kraus et al., 2013; Howard et al., 2014). Such a response reflects a cellular-level accumulated error in timing from the outset of the to-be-timed interval similar to the scalar property in behavioral experiments of interval timing (Gibbon and Church, 1984; Gibbon et al., 1984). This paper suggests a possible bridge between the cellularlevel, experimentally measured, proportionality relationship between the spread of the time field and the time of peak activity of the time cell (Pastalkova et al., 2008; MacDonald et al., 2011; Kraus et al., 2013; Howard et al., 2014) and the scalar property of interval timing. The linear relationship between the peak time and the spread of the Gauss-like, i.e., the error of time estimation increases linearly with the tobe-timed interval, which has been measured in behavioral experiments, such as the peak interval procedure (Buhusi and Meck, 2005, 2006; Buhusi et al., 2006, 2009; Buhusi and Oprisan, 2013).
Similarities and Differences Between This Novel Population-Based Topological Map of the Hippocampus and Other Timing Models. This new model of temporal memory storage in the hippocampus is based on two core hypotheses: (1) the existence of a spatially ordered memory of temporal durations (topological map) in the hippocampus, and (2) the time cells form a temporal basis for time perception. This model of the long-term memory block could also be included in more comprehensive timing models, such as the Scalar Expectancy Theory (SET) (Gibbon, 1977; Church, 1984; Church and Broadbent, 1990, 1991; Church et al., 1994, 1998; Gibbon et al., 1997), Striatal Beat Frequency (SBF) (Buhusi and Meck, 2005, 2009, 2010; Oprisan and Buhusi, 2011, 2013, 2014; Buhusi and Oprisan, 2013; Buhusi et al., 2016; Oprisan et al., 2018), the Behavioral theory of Timing (BeT) (Killeen and Fetterman, 1988, 1993; Bizo and White, 1994), or the Learning-to-Time (LeT) model (Machado, 1997; Machado and Silva, 2007; Machado et al., 2007). Without going into an extensive literature review of the SET model (see Gallistel, 1990; Gibbon, 1991; Church, 2003 and references therein), we only briefly mention here the key functional building blocks of the SET model that other theoretical models of time perception preserved and expanded upon. The SET model postulates the existence of an internal clock composed of three blocks: a pacemaker-accumulator, a memory, and a comparator. The role of the pacemaker is to generate clock pulses that are added up by the accumulator block. The accumulator is reset at the beginning of every trial, presumably through a dopamine-mediated mechanism. The long-term memory block stores the value of the accumulator at the reinforcement time in each trial. After a large number of trials, the long-term memory contains a Gaussian distribution of reinforcement times approximating the criterion time. According to the SET model, the decision whether or not to respond in a test trial is determined by the ratio between a randomly drawn value of the criterion time from its multiple, Gauss distributed, copies stored in the long-term memory and the current value of the accumulator. When the ratio computed by the comparator block crosses a given threshold, the animal changes its response from a low to a high rate. Our previous topological map model of the hippocampus also stored a set of reinforcement times in the long-term memory (Oprisan et al., 2018). Compared to SET, the novelty of the topological map was the hypothesis of a spatially ordered structure of the hippocampus, which allowed us to explain the experimental results on hippocampus lesions.

Both BeT and LeT models require three elements: a series of states, a series of operant responses, and a set of associative links connecting the states to the operant responses. It is convenient to conceptualize the transitions between states as being driven by a pacemaker block ("even though the mathematical models do not strictly require a biological pacemaker that emits pulses" Killeen and Fetterman, 1993). In the BeT and LeT models, a certain number of states may be required to produce an operant response. Although it is "unclear how states relate to measurable behavior or what their neural basis is" (Machado et al., 2007), it is assumed that they are related to behavioral responses through associative weights that have a natural rate of decay and a 
reinforcement-dependent rate of increase. There is a similarity between our proposed population-based topological map model of the hippocampus and the LeT model: both use a distributed set of weights. In the LeT model, the weights of the associative links connect states to the operant response, whereas in our computational model the weights represent contributions of time cells to the average time field. In the model we present here, we preserved the topological map from Oprisan et al. (2018) and replaced the actual reinforcement times by the weights of the corresponding time cells. This improvement allowed us to define a learning rule for the weights in order to minimize the error signal, which is proportional to the temporal distance between the time of the peak activity for a given time cell and the to-be-learned criterion time.

\section{TEMPORAL MAPS IN THE HIPPOCAMPUS GENERATED BY A POPULATION OF "TIME CELLS"}

\subsection{A Population-Based Model for the Topological Map of the Hippocampus}

While we successfully modeled the hippocampus lesions with the previously proposed topological maps (Oprisan et al., 2018), the model was not well-connected with neurobiology. Like SET model, it assumed that the hippocampus simply records the reinforcement times with the added twist of a spatially ordered temporal map with increasingly longer durations stored toward the dorsal side of he hippocampus. Additionally, the topological map model assumed that the distribution of memorized times is Gaussian, with more memory cells holding reinforcement times closer to the criterion time. This is a heuristic assumption consistent with the SET model (see Church, 1984; Gibbon and Church, 1984; Gibbon et al., 1984, 1988; Brunner et al., 1997) hypothesis that at the reinforcement time each trials stores in the hippocampus a slightly different value of the to-be-memorized criterion time. In this study, we suggested a novel approach to the existing topological map model. In order to preserve the ability of the new model to match experimental data on hippocampus lesions, we still assumed that a spatially ordered (topological) map exists in the hippocampus. However, in this new model a memory cells no longer stores a single value, i.e., the reinforcement time $t_{n}$, but rather stores a pointer to an entire object, i.e., a time cell whose activity peaks at the specific time $t_{n}$. Therefore, here we suggest a new model of the long-term memory based on a possible connection between the hippocampus topological maps and a population of time cells.

Model Constraint: Scale Invariance of Time Perception. The experiments on time perception seem to suggest that the time perception error increases linearly with the to-be-timed duration, which is referred as the scalar property (Buhusi et al., 2017; Daniels and Sanabria, 2017). Scalar property has been found in episodic memory (Glenberg et al., 1980; Howard et al., 2008), peak interval timing (Lewis and Miall, 2009), and conditioning (Balsam and Gallistel, 2009). At the same time, scalar property is shared across different species from mice (Malapani and Fairhurst, 2002; Buhusi et al., 2009) to humans (Rakitin et al.,
1998). As a result, every time perception model must include an explanation for the origin of the scale invariance.

Model Constraint: Peak Time Shift After Hippocampus Lesions. Hippocampus lesion experiments showed that dorsal hippocampal (DH) lesions induced leftward shifts in peak times (Tam et al., 2015), i.e., toward shorter durations, whereas ventral hippocampal (VH) lesions produced opposed peak shift (Yin and Meck, 2014). Based on the above-cited and similar experimental results, we recently suggested a topological map model of the hippocampus (Oprisan et al., 2018). Briefly, the reinforcement times centered around the desired criterion time learned during successive conditioning trials were modeled as Gaussian random variables. The Gaussian distribution hypothesis was based on existing experimental observations and the justification from the influential SET model (see Church, 1984; Gibbon and Church, 1984; Gibbon et al., 1984, 1988; Brunner et al., 1997). SET assumes that the to-be-learned duration is the result of many training trials that add up to a Gaussian-like response (see Figure 1A). Since lesion experiments suggested a very specific, ordered, arrangement of the memorized durations in the hippocampus (see Tam and Bonardi, 2012a,b; Tam et al., 2013, 2015; Yin and Meck, 2014), we modeled the hippocampus as a spatially ordered map that stores a Gaussian-like distribution of reinforcement times. That topological map model of time perception assumed that the hippocampus serves as a memory storage for elapsed durations with the shorter durations orderly stored toward the ventral side and progressively longer durations stored toward the dorsal side (Oprisan et al., 2018). Such a spatial localization (topological map) of reinforcement times allowed us to both (1) explain experimentally observed effects of hippocampus lesions on interval timing and (2) predict mathematically the relationship between the width and the peak location of the Gaussian-like output function. Without repeating the derivations from Oprisan et al. (2018), we previously showed that the relationship between the width and the peak duration of the output function after the lesions matches the pre-lesion relationship (Oprisan et al., 2018). In other words, our topological map model of the hippocampus preserved the properties of the distribution of memorized durations and only shifted the peak responses proportional to the lesion size and its location relative to the median line of the hippocampus (Oprisan et al., 2018).

Hypothesis 1: Temporal Boundary. First, we need to explain how the time cells of the hippocampus know to cover the entire to-be-timed duration. For this purpose, we hypothesize that the mechanism is similar to the one used by the "place cells" of the hippocampus, i.e., place cell firing depends on the position of environmental boundaries (O'Keefe and Burgess, 1996). Similarly, during peak-interval procedure, the mouse learned the "boundaries" of the temporal duration through a series of reinforcement trials. In our current implementation, the temporal boundary of the time cells is three times the criterion time with time cells uniformly distributed over the entire duration.

Hypothesis 2: Coincidence Detection Dynamically Adjusts Time Cell Firing Rates. The second assumption of our newly proposed mechanism for generating a Gaussian-like and scale 

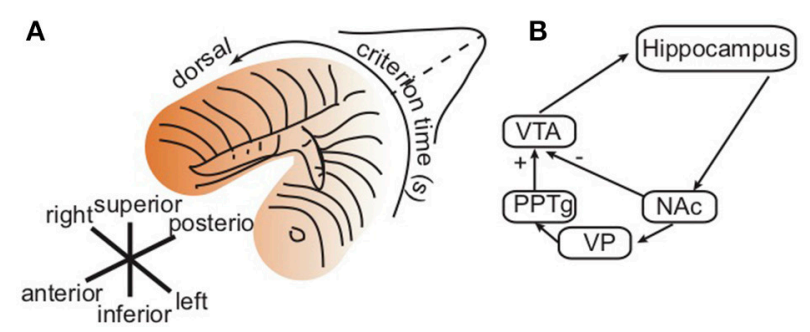

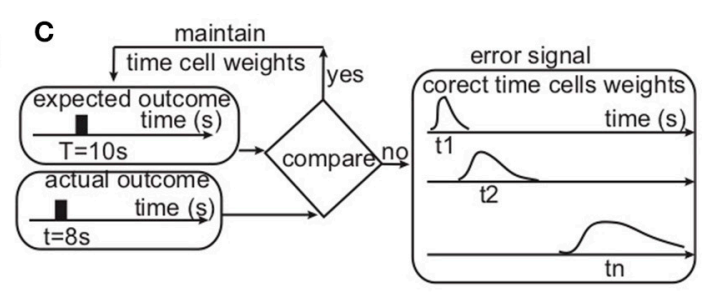

FIGURE 1 | Hippocampal topological map and basal ganglia-hippocampal loops. (A) Consistent with hippocampus lesions experiments, the sketch of the hippocampus with color shading suggesting that shorter durations are stored in the ventral area and longer durations orderly stored in the dorsal area. (B) Simplified basal ganglia-hippocampal loops with direct projection to the nucleus accumbens (NAc), which performs a decision task by comparing the predicted and the actual outcomes of behavior. If the outcome is as expected, the result is an inhibition ("-") of ventral tegmental area (VTA). Otherwise, an error correction loop that includes the ventral pallidum (VP) and the pedunculopontine nucleus (PPTg) excites ("+") VTA. (C) Symbolic representation of computations performed by the basal ganglia-hippocampus loops in order to correct the activity of time cells such that the average time field peaks around the to-be-memorized criterion time $T$. An error signal proportional to the distance between the expected peak time $T$ and the actual peak times $t_{1}, t_{2}, \ldots, t_{n}$ of individual time cells changes the weights of the contribution of the individual time cells.

invariant average time field is that dopamine modulation could enhance the activity of certain time cells during reinforcement trials and depressed the activity of others. In order to know which weights to strengthen/weaken, we rely on the existence of a coincidence detector mechanism, such as the one attributed to striatum (Houk, 1995; Houk et al., 1995; Parent and Hazrati, 1995a,b; Harrington et al., 1998; Atallah et al., 2004; Jin et al., 2009; Buhusi et al., 2016), that selectively enhances the activity of those time cells that fire close to the to-be-timed duration. Additional evidence of a possible involvement of the striatum as a coincidence detector is that the cortico-striatal system is involved in "habit" or "procedural" learning. The dopaminergic projections to striatum also support the hypothesis of a modulatory feedback effect that enhances desirable behaviors and suppresses others (Schultz, 2002). It has been suggested that long-term potentiation/depression could reliably modulate the synaptic weights in cortico-striatal loops (Teki et al., 2012). Such dopamine-mediated reinforcement signals could fine-tune the synaptic weights based on repeated trails until the correct criterion time is learned ( $\mathrm{Gu}$ et al., 2011; Jones and Jahanshahi, 2011). The hippocampus stores representations of individual experiences and seems to carry out a completely different type of memory function than the striatum (Packard et al., 1994; Canal et al., 2005), although some view the two systems as complementary (Atallah et al., 2004). Figure 1B shows a sketch of a possible neurobiological network involving time cells in the hippocampus, the striatum coincidence detector and the dopaminergic reinforcement.

Possible Basal Ganglia-Hippocampus Loops. Our suggested simplified network (Figure 1B) uses well-known direct (Voorn et al., 2004) and indirect (Christakou et al., 2004) anatomical connections between the hippocampus and the striatum (see Thierry et al., 2000 for an extensive review of existing pathways). For example, there are known GABAergic projections from the basal ganglia nuclei, the substantia nigra pars reticulata, and the internal part of the globus pallidus to pontine nucei (Parent and Hazrati, 1995a,b). In turn, cholinergic projections from the pedunculopontine nucleus directly (Datta et al., 1998; Silkis, 2008) and indirectly (through thalamic nucleus reuniens Vertes, 2001; McKenna and Vertes, 2004) control the activity of the hippocampus. Among many other identified neural loops, neurons of the CA1 hippocampus area project mainly to the nucleus accumbens (NAc) of the striatum. This nucleus projects into the thalamic nuclei of the middle line through the ventral pallidum, and these thalamic nuclei project back into the NAc and hippocampus (Groenewegen et al., 1999).

A simplified loop (Penner and Mizumori, 2012) that involves a direct projection from the hippocampus to the nucleus accumbens (NAc) of the ventral striatum seems to perform a coincidence detection task by comparing the predicted and the actual outcomes of behavior and either (1) inhibits (marked by “-” in Figure 1B) ventral tegmental area (VTA) if the action matches the predicted behavior, or (2) excites ("+") VTA in the case of a mismatch. One possible realization of such an indirect path is via ventral pallidum (VP) and the pedunculopontine nucleus (PPTg) (Penner and Mizumori, 2012). One possible effect of this excitatory input to VTA is to bias it more closely to a bifurcation point where is more sensitive to subsequent reward information (see Figure 1B).

We assume here that the neurobiologically realistic structure sketched in Figure 1B could perform the following computations. Initially, the hippocampus long-term memory stores an arbitrary distribution of weights associated to time cells that peak in the range of the to-be-memorized temporal duration. At the reinforcement time, the striatum coincidence detector updates the weights according to Equation (3), i.e., the time cells that peak closer to the reinforcement time have larger weights compared to those that peak farther. This modulation of time cell activity produces a population response that peaks around the reinforcement time. The weight computation is also flexible enough to ensures the shift of the entire population response to a new reinforcement time. For example, given that the expected outcome should peak at the criterion time $T=10 \mathrm{~s}$ whereas the actual response in a given trial was at $t=8 \mathrm{~s}$, an error signal is generated (see Figure 2). The heuristic mechanism connecting the time of the peak activity of a time cell with the desired behavioral response uses the error signal to modulate the 

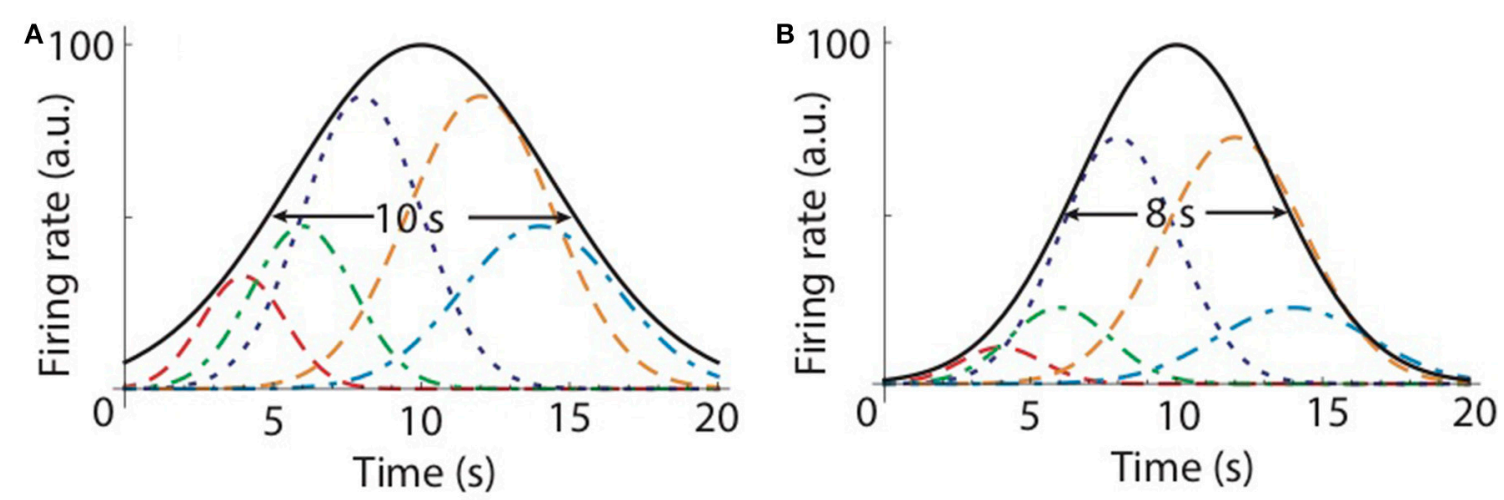

C

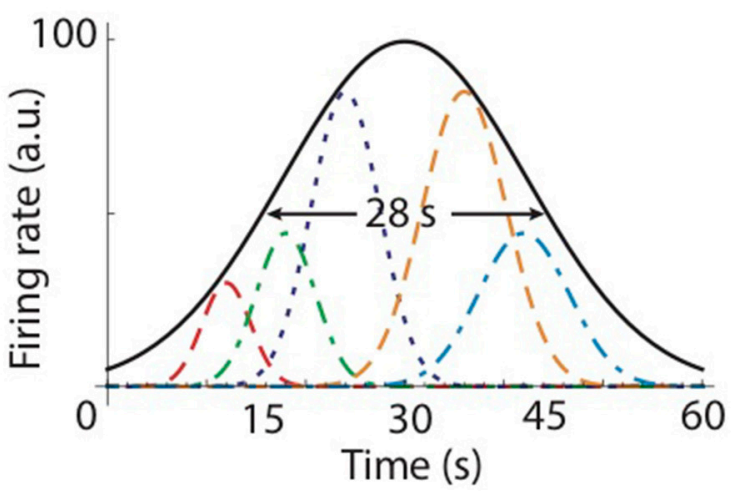

。

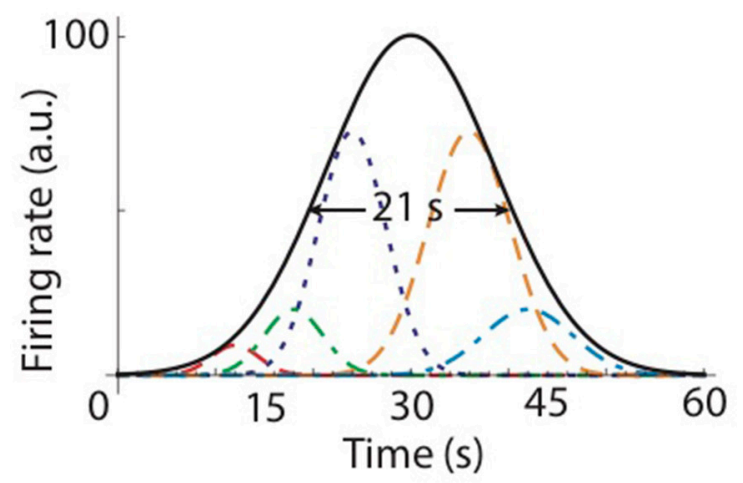

FIGURE 2 | A population of time cells could learn the temporal map for the hippocampus. (A) Firing rate curves for five representative time cells that peak at $4 \mathrm{~s}$ (red dashed line), $6 \mathrm{~s}$ (green dashed-dotted line), $8 \mathrm{~s}$ (blue dotted line), $12 \mathrm{~s}$ (orange dashed line), and $14 \mathrm{~s}$ (slate blue dashed-dotted line), respectively. Starting from an initially uniform distribution of weights, the first learning trial adjusts the weights to $0.15,0.22,0.4,0.4$, and 0.22 , respectively. (B) Based on Equation (3), the second trial further adjusted the weights to $0.02,0.05,0.16,0.16$, and 0.05 , respectively. The envelope of the population of time cells (black continuous line) shows that the peak is around the to-be-learned criterion time $T=10 \mathrm{~s}$ and the envelope becomes narrower as the number of trials increases. (C) For the criterion time of $T=30 \mathrm{~s}$, only some representative firing rate curves are shown for $12 \mathrm{~s}$ (red dashed line), $18 \mathrm{~s}$ (green dashed-dotted line), $24 \mathrm{~s}$ (blue dotted line), $36 \mathrm{~s}$ (orange dashed line), and $42 \mathrm{~s}$ (slate blue dashed-dotted line), respectively. The envelope of the population (black continuous line) peaks at $T=30 \mathrm{~s}$. (D) The envelope for the second trial is narrower and its width for a criterion time $T=30 \mathrm{~s}(\mathbf{C}, \mathbf{D})$ is approximately three times larger compared to width for $T=10 \mathrm{~s}(\mathbf{A}, \mathbf{B})$.

activity of the time cell proportional to its temporal distance to the expected criterion time.

\section{THE MODEL}

Based on experimental evidences regarding the existence and the properties of the time cells (Pastalkova et al., 2008; MacDonald et al., 2011; Kraus et al., 2013; Howard et al., 2014), we modeled the activity of each time cell with a Gaussian firing rate curve with the width modulated by its peak time:

$$
a_{n}\left(t_{n}, \sigma_{n}\right)=A_{n} e^{-\frac{\left(t-t_{n}\right)^{2}}{2 \sigma_{n}^{2}}},
$$

where we assumed for simplicity that the standard deviation $\sigma_{n}$ is proportional to the peak time $t_{n}$ (see MacDonald et al., 2011 for experimental support of this assumption) and $A_{n}$ is the maximum firing rate of the time cell with the peak activity at $t_{n}$.

In agreement with the experimental data (Pastalkova et al., 2008; MacDonald et al., 2011; Kraus et al., 2013; Wang et al.,
2015), we assumed that there is a certain, finite, number $N$ of time cells that span the entire range of durations required by the behavioral experiment. The error signal (see Figure 1B) modulates the contribution of the individual time cells until the average time field matches as closely as possible the expected outcome. The average time field is determined by the weighted average of the time fields of the $N$ time cells:

$$
a\left(T_{a v g}, \sigma_{a v g}\right)=\sum_{k=1}^{N} A_{k} e^{-\frac{\left(t-t_{k}\right)^{2}}{2 \sigma_{k}^{2}}},
$$

where $N$ is the number of time cells allocated to the current timing task and $A_{k}$ is the amplitude (weight) associated to an individual time cell. For example, if the expected outcome is $T=10 \mathrm{~s}$, then ideally all time cells would have zero weights $A_{k}=0$, except for the cells that peak at $t_{k}=T=10 \mathrm{~s}$. We mathematically modeled the error signal needed in Figure 1C as the difference between the expected outcome $T$ and the peak time 
$t_{k}$ of each time cell:

$$
\operatorname{err}_{k}=\left|T-t_{k}\right|+\epsilon,
$$

where $\epsilon$ is a very small positive number that helps us avoid a mathematical singularity for the learning rule (Equation 4). At the same time, a small positive $\epsilon$ mimics the ubiquitous biological noise. The learning rule is:

$$
A_{k} \leftarrow A_{k} / \text { err }_{k},
$$

where $\leftarrow$ indicates that after each learning trial the current weights $A_{k}$ are replaced by $A_{k} / e r r_{k}$. The effect of the above learning rule is that time cells that peak at $t_{k}$ further from the expected outcome $T$ would be stronger depressed compared to those closer to the expected outcome. The weights $A_{k}$ are stored in a spatially ordered (topological map) memory register that mimics the hippocampal structure (see Figure 1).

\section{RESULTS}

\subsection{Theoretical Predictions Regarding the Scalar Property}

There are two distinct contributions to the width of the average time field: (1) the intrinsic properties of individual time field of each time cell, and (2) the global weights learning algorithm.

Time Cell Effect on the Width of the Average Time Field. The half-width of an individual time field of a single cell that peaks at $t_{k}$ is determined by Equation (1), i.e.,

$$
e^{-\frac{\left(T-t_{k}\right)^{2}}{2 \sigma_{k}^{2}}}=1 / 2
$$

which gives width $_{\text {cell }}=\left|T-t_{k}\right|=\sigma_{k} \sqrt{2 \ln 2}$ as shown in Figure 3.

Weights Learning Effect on the Width of the Average Time Field. Even if all time cells were to peak precisely at only one moment in time with no time field width, the average time field still would have a spread determined solely by the learning rule. A time cell that peaks at $t_{k}$ which exactly overlaps with the criterion time $T$ has the maximum possible weight, i.e., $w_{\max }=\frac{1}{\left|T-t_{k}\right|+\epsilon}=$ $\frac{1}{\epsilon}$ (see Figure 3). To find out the half-width of the average time field due to the weights changing algorithm we need to find the peak activity time $t_{p} \neq T$ when the weight $w_{t_{p}}$ is half the maximum weight. Since $w_{t_{p}}=\frac{1}{\left|T-t_{p}\right|+\epsilon}$, the width of the average time field due to the learning rule imposed on the weights is:

$$
\text { width }_{\text {learn }}=\left|T-t_{p}\right|=\epsilon .
$$

As we notice from Figure 3A, it is possible that the intrinsic width of every time field ( width $_{\text {cell }}$ ) is larger than the learning spread width learn. In this case, the width of the average Gaussian time field is determined by the time cell properties. Since the time fields of individual time cells obey the scalar property, it results that the average time field will also obey the same property with exactly the same coefficient of variation as the time cell that is closest to the criterion time. It is also possible to have very narrow time cell fields such that width $_{\text {cell }}<$ width learn $_{\text {(see Figure 3B). In }}$ this case, the width of the average time field is entirely determined by the constant $\epsilon$ of the learning rule and the scalar timing is no longer observed. Therefore, depending on the ratio between the intrinsic variance $\sigma_{k}$ of time cell field and the learning rule constant $\epsilon$, it is possible to observe either scalar timing or average time fields of constant width. In practice, we can always select the learning rule constant $\epsilon$ such that $\sigma_{k} \sqrt{2 \ln 2}>\epsilon$ for all criteria such that the average time field obeys scalar property.

\subsection{The Convergence of the Learning Algorithm}

To emphasize that the width of the individual fields is also proportional to the peak time of the corresponding cells we only showed symmetrically distributed time fields in Figure 2. However, the learning algorithm from Equation (4) converges to the desired criterion time $T$ because the learning rule always gives higher weights to cells firing closer (in time) to $T$. Therefore, regardless the initial distribution of weights, they will be repeatedly changed to minimize the error signal from Equation (3), i.e., to reduce the temporal distance of the average time field to the desired criterion time. The weight recalculation only depends on the peak time of the individual time cell $t_{k}$ and the criterion time $T$. As a result, regardless the distribution of the peak times of time cells (symmetric or not with respect to the criterion time), their weights will always be adjusted to favor cells closer to the criterion time $T$. At the same time, a sparse and asymmetric distributed of time cells could influence the accuracy of timing. For example, for a criterion time of $T=10 \mathrm{~s}$ it could be that only the following time cells are available: $6,8,12,20$, and 30 $\mathrm{s}$, respectively. In this case, although the weights are still correctly calculated to favor the closest time cell to the criterion time of $T=10 \mathrm{~s}$, the average time field will peak around $8 \mathrm{~s}$, which leads to a large error compared to the actual criterion time $T=10 \mathrm{~s}$. This example suggests that the accuracy of the learning algorithm is determined by the temporal distance of the closest time cell to the criterion time, i.e., $\operatorname{err} r_{\text {timing }}=\min \left\{\left|T-t_{k}\right|\right\}$.

\subsection{Storing Multiple Criteria With the Population-Based Topological Map Model}

After a set of training trials, the weights stabilize such that the model responds sharply to a criterion time of $T_{1}=10$ s, i.e., the weights of the time cells near $T_{1}=10 \mathrm{~s}$ are the largest and they decay with the temporal distance from the desired criterion time. In our implementation of the learning rule given by Equation (4), the weight for a time cell with its peak activity at the desired criterion time $t_{1}=10 \mathrm{~s}$ is $w_{t_{1}}=\frac{1}{\left|T_{1}-t_{1}\right|+\epsilon}=\frac{1}{\left|T_{1}-10 s\right|+\epsilon}=\frac{1}{\epsilon}$. During the same training trial, a cell that peaks at $t_{2}=100 \mathrm{~s}$ has a weight of $w_{t_{2}}=\frac{1}{\left|T_{1}-t_{2}\right|+\epsilon}=\frac{1}{\left|T_{1}-100 s\right|+\epsilon}=\frac{1}{90+\epsilon}$. In other words, the weight of the time cell firing at $100 \mathrm{~s}$ is about 90 times weaker $\left(\frac{w_{t_{2}}}{w_{t_{1}}}=\lim _{\epsilon \rightarrow 0} \frac{\epsilon}{90+\epsilon} \approx \frac{1}{90}\right)$ than the one firing at the correct criterion time $T_{1}=10 \mathrm{~s}$.

When a new set of training trials starts for a different criterion time $T_{2}=100 \mathrm{~s}$, it finds the highest weight $w_{t_{1}}=\frac{1}{\epsilon}$ and changes it to $w_{t_{1}} \leftarrow \frac{w_{t_{1}}}{e r t_{1}}=\frac{1}{\epsilon} \frac{1}{\left|T_{2}-t_{1}\right|+\epsilon}=\frac{1}{\epsilon(90+\epsilon)}$. Similarly, at the start of 

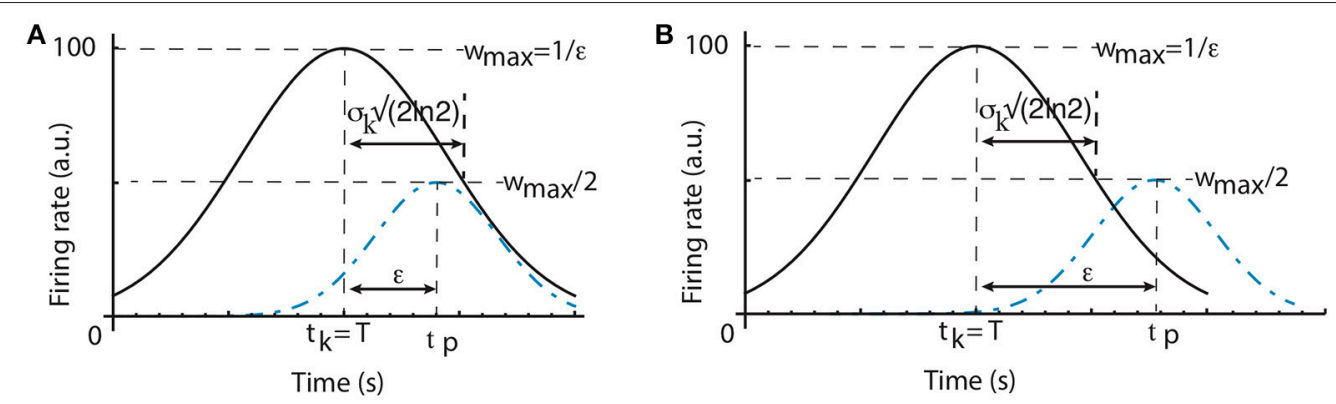

FIGURE 3 | Scalar property predictions. The half-width of the time field of a time cell that peaks at $t_{k}$, which coincides with the criterion time $T$, is width cell $=\sigma_{k} \sqrt{2} \ln 2$ (A). The width of the average time field determined by the learning algorithm is given by the peak time $t_{p}$ for $w_{h i c h} w_{t_{p}}=\frac{1}{2} w_{m a x}=\frac{1}{2 \epsilon}$ (regardless the criterion time). As a result, width $_{\text {learn }}=\epsilon$. If all individual time cell fields are wide enough such that $\sigma_{k} \sqrt{2 \ln 2}>\epsilon$, then the average time field obeys the scalar property (A). Otherwise, if the time fields of individual time cells are very narrow, then the width of the average time field is only determined by the parameter $\epsilon$ of the learning algorithm (B).

a trial for a new criterion time $T_{2}$ the initial weight of $w_{t_{2}}=\frac{1}{90+\epsilon}$ will be changed to $w_{t_{2}} \leftarrow \frac{w_{t_{2}}}{e r r_{t_{2}}}=\frac{1}{90+\epsilon} \frac{1}{\left|T_{2}-t_{2}\right|+\epsilon}=\frac{1}{\epsilon(90+\epsilon)}$. As we notice, the two weights for the two memorized criteria $T_{1}=10 \mathrm{~s}$ and $T_{2}=100 \mathrm{~s}$ remain the largest weights among all memorized values in the hippocampus without requiring any additional counter mechanism to distinguished between $T_{1}$ and $T_{2}$.

\subsection{Numerical Results}

We numerically tested our population-based topological map model and the above learning rule given by Equation (4). We started all numerical simulations with a population of time cells that uniformly covered a time interval that included the criterion time. The range of the peak times covered by the time cells was three times the criterion time. Initially, all time cells have the same maximum firing rate (weight), although we tested that the model works with an arbitrary initial distribution of weights. We tested the model with a variable number of time cells $(N=10$, 100 , and 1,000) and found no difference among the respective average time fields. The criteria tested were $T=10 \mathrm{~s}$ (see Figures 2A,B) and $T=30 \mathrm{~s}$ (see Figures 2C,D). In the example shown in Figures 2A,B, we only plotted the firing rate curves of five representative time cells that peak at $4 \mathrm{~s}$ (red dashed line), $6 \mathrm{~s}$ (green dashed-dotted line), $8 \mathrm{~s}$ (blue dotted line), $12 \mathrm{~s}$ (orange dashed line), and $14 \mathrm{~s}$ (slate blue dashed-dotted line), respectively. After the first learning trial, the weights associated with the time cells were set inversely proportional to the their distance from the to-be-learned criterion time, i.e., $T=10 \mathrm{~s}$. Based on our learning rule, for the cells shown in Figure 2, the weights after the first trial became $0.15,0.22,0.4,0.4$, and 0.22 , respectively (Figure $2 \mathrm{~A}$ ). This shows that the two closest time cells to the to-be-learned criterion time at $8 \mathrm{~s}$ and $12 \mathrm{~s}$ have the highest weights and all the other weights decrease as the corresponding time cell is farther from the criterion time $T=$ $10 \mathrm{~s}$. The second trial further adjusted the weights to $0.02,0.05$, $0.16,0.16$, and 0.05 , respectively (Figure $2 B$ ). The envelope of the population of time cells (black continuous line in Figure 2) shows that the peak is around the to-be-learned criterion time $T$ and the envelope becomes narrower as the number of trials increases. For example, for $T=10 \mathrm{~s}$ (Figures 2A,B), the halfwidth of the envelope is $10 \mathrm{~s}$ after the first trial and $8 \mathrm{~s}$ after the second. Since the weights associated with the contribution of different time cells to the average time field decrease after each trail as a power law, we predict that the width of the average time field will eventually equal the width of the closest time cell to the actual criterion time. We also tested different criteria, such as $T=30 \mathrm{~s}$ (Figures 2C,D), and found that the half-width of the envelope is indeed approximately three times its value for $T=10 \mathrm{~s}$. Therefore, this topological map with time cells and the learning rule given by Equation (4) generates average time fields that follow the scalar property of interval timing.

\section{DISCUSSION}

One of the first and most prominent timing paradigms is the counter model in which a counter keeps track of how many "clock ticks" accumulated since the presentation of a certain salient stimulus that reset the counter (Gibbon, 1977). Later on the counter model was implemented as a drift-diffusion processes with the "clock" represented by some regularly firing neurons (Simen et al., 2011b; Luzardo et al., 2013). A unified model of conditioning and timing that is at its core a driftdiffusion model has been recently developed (Luzardo et al., 2017).

Difficulties of Counter-Based Models. One difficulty of the counter models (Gibbon, 1977; Rakitin et al., 1998; Gallistel and Gibbon, 2000) is that they cannot time multiple intervals. For example, in conditioning experiments that rewards the subject either for the first response after a short delay, say $10 \mathrm{~s}$, or the first response after a long delay, say $100 \mathrm{~s}$, the responses were bimodal when the reward is omitted (Catania and Reynolds, 1968; Leak and Gibbon, 1995). However, based on the counter theory, unless the counter model uses different counters for different durations, the output is unimodal with a peak around the average of the two durations. On the other hand, using different counter requires additional information, which is not experimentally available, to identify 
which counter must be updated (Gallistel, 2007; Machado and Silva, 2007).

Difficulties of Ramping Firing Models. Although we used firing rate models to mimic the activity of time cells and to match experimental observations, our population-based time field model does not have the limitations of other firing rate-based models. For example, ramping firing models (Simen et al., 2011b), are limited by the time scale of the leaky integrators. Recently, theoretical approaches suggested that persistently firing neurons (Egorov et al., 2002) could be used to construct time constants up to a few thousand seconds (Tiganj et al., 2015). While a significant improvement, the challenge for the ramping firing model still remains when it comes to covering interval timing processes on a much longer time scale (Howard et al., 2014). A recent solution proposed in Simen et al. (2011a) suggested using a chain of leaky integrators with a decreasing sequence of intrinsic time constants to implement a feedback-based integrator.

Similarities With SET and LeT Models. Similar to the SET model, the long-term memory of our previous topological model of the hippocampus (Oprisan et al., 2018) stored a set of reinforcement times, which presumably represent a Gaussian distribution with its peaks around the to-be-learned criterion time. However, we departed from the SET model by assuming in our topological model that the durations are stored in a spatially ordered memory register with short durations stored ventrally and longer durations more dorsal (Oprisan et al., 2018). Such a topological organization was inspired by experimental observations on peak interval shift due to hippocampus lesions. While conceptually straightforward, one drawback of the topological map model is the nature of what is actually stored in the hippocampus. Computational models can store anything, including a floating point number that is the actual reinforcement time of an individual trial (like in SET model). In this paper we suggested a more realistic model of the topological map of the hippocampus by assuming that the memory register actually stores weights associated to individual time cells. This new population-based topological map model of the hippocampus is an improvement over the previous model (Oprisan et al., 2018) in many respects: (1) the properties of the average time field, such as the peak time and width, are directly related to the time field of the time cells, (2) the learning mechanism of the weights ensures that the average time field peaks around the to-be-learned criterion time, and (3) long-term potentiation/depression mechanisms could be invoked for adjusting the weights. The replacement of the memory register content with the weights of the time cells instead of actual reinforcement time is a departure from the SET model and a similarity point with the LeT model. However, in contrast to LeT, in our model the weights are associated to physical time cells of the hippocampus and serve for computing the average time field. At the same time, this new model of a population-based topological map of the hippocampus with time cells is different from LeT weight adjustment model: (1) we did not consider any intrinsic decay rates of the weights as in LeT, and (2) we did not directly relate the weight strengthening to the reinforcement time of individual trials.

Similarities With Microstimuli Model. Recently, the microstimuli model (Ludvig et al., 2008) suggested that when a stimulus is presented, it leaves a slowly decaying memory trace, which is encoded by a series of temporal receptive fields. The theory of microstimuli also postulates that the traces with long latency peaks have greater dispersion. Experimental evidences for microstimuli theory have been found in basal ganglia (Adler et al., 2012). They found Gaussian-like post-stimulus time courses (similar to those describing the temporal field of time cells in our model given by Equation 1) in medium spiny neurons recording from the putamen. Similar evidences of the existence of Gauss-like responses were found in caudate nucleus (Jin et al., 2009). While Gauss-like structures of temporal fields have been used by many models from the early SET (Church, 1984; Gibbon and Church, 1984; Gibbon et al., 1984, 1988; Brunner et al., 1997) to the more recent microstimuli (Ludvig et al., 2008), or the time cell temporal field (Pastalkova et al., 2008; MacDonald et al., 2011; Kraus et al., 2013; Wang et al., 2015), our topological map model only apply to the hippocampus. Interval timing has always been thought to involve multiple, complementary, mechanisms served by distributed neural networks. One such distinct mechanism could be the basal ganglia-based microstimuli model.

Here we suggested a novel, population-based, model that uses experimentally verified results regarding the time cells of the hippocampus. The activity of a time cell (1) peaks at a specific time during a timing task, and (2) the firing field width increases with the peak time (Pastalkova et al., 2008; MacDonald et al., 2011; Kraus et al., 2013; Howard et al., 2014). We suggested a possible time perception mechanism which assumes that (1) the hippocampus stores a spatially organized (topological) map of durations (Oprisan et al., 2018) with the added novelty that (2) a memory location in the hippocampus actually stores a pointer to a whole object, which is a time cell defined by the timing of its peak activity and the width of its timing field. Previously, the topological map model introduced in Oprisan et al. (2018) only stored reinforcement time values in a spatially ordered register with smaller durations mapped toward the ventral side and larger durations stored toward the dorsal area of the hippocampus. Such a topological organization accounts for the experimentally observed effect of hippocampus lesions (Yin and Meck, 2014; Tam et al., 2015). While the existence of such topological maps has been supported by experiments, we suggested here a more flexible model that instead of storing actual temporal durations it stored a pointer to a whole object, i.e. a time cell. At the computer implementation level, the topological map actually stores the maximum amplitude (the weight) of the Gaussian firing rate associated to a given time cell. The learning rule for the weights given by Equation (4) accounts for the contribution of individual time cells to the average time field and implements a distancedependent connectivity, which has experimental support (Perin et al., 2011). Based on this newly proposed learning rule, we found that the average time field based on the topological map of a population of time cells preserves both the Gaussian-like structure and the scalar property of the individual time cell fields. 
One obvious implication of this topological map mode with time cells is that the properties of the average time field, i.e., the accuracy of timing and the scalar property, are directly determined by the similar properties of the time cells. As the experiments showed, the firing field of individual time cells is Gaussian and its width scales with the peak time. This cellularlevel scalar property is inherited by the average time field, which is a weighted average of individual time fields. Additionally, the shape of the average temporal field is also influenced by the learning rule. Here we adopted a weight adjustment rule based on the temporal distance from the criterion time to the peak time of each individual cell. While experimental support exists for such a distance-based weight computation, other models are possible. Therefore, new experimental data that manipulate the activity of time cells are necessary in order to uniquely identify the learning rule.

This model is only a small piece of a wider picture which attempts to explain how time perception forms in the brain. As it has been suggested, it is likely that there are multiple mechanisms for time perception implemented by a set of distributed neural networks working together toward this goal.

\section{REFERENCES}

Adler, A., Katabi, S., Finkes, I., Israel, Z., Prut, Y., and Bergman, H. (2012). Temporal convergence of dynamic cell assemblies in the striato-pallidal network. J. Neurosci. 32, 2473-2484. doi: 10.1523/JNEUROSCI.4830-11.2012

Atallah, H. E., Frank, M. J., and O'Reilly, R. C. (2004). Hippocampus, cortex, and basal ganglia: insights from computational models of complementary learning systems. Neurobiol. Learn. Mem. 82, 253-267. doi: 10.1016/j.nlm.2004. 06.004

Balsam, P. D., and Gallistel, C. R. (2009). Temporal maps and informativeness in associative learning. Trends Neurosci. 32, 73-78. doi: 10.1016/j.tins.2008.10.004

Bizo, L. A., and White, K. G. (1994). The behavioral theory of timing: reinforcer rate determines pacemaker rate. J. Exp. Anal. Behav. 61, 19-33. doi: 10.1901/jeab.1994.61-19

Brunner, D., Fairhurst, S., Stolovitsky, G., and Gibbon, J. (1997). Mnemonics for variability: remembering food delay. J. Exp. Psychol. Anim. Behav. Process. 23, 68-83.

Buhusi, C. V., Aziz, D., Winslow, D., Carter, R. E., Swearingen, J. E., and Buhusi M. C. (2009). Interval timing accuracy and scalar timing in C57BL/6 mice. Behav Neurosci. 123, 1102-1113. doi: 10.1037/a0017106

Buhusi, C. V., and Meck, W. H. (2005). What makes us tick? Functional and neural mechanisms of interval timing. Nat. Rev. Neurosci. 6, 755-765. doi: $10.1038 / \mathrm{nrn} 1764$

Buhusi, C. V., and Meck, W. H. (2006). Time sharing in rats: a peakinterval procedure with gaps and distracters. Behav. Process. 71, 107-115. doi: 10.1016/j.beproc.2005.11.017

Buhusi, C. V., and Meck, W. H. (2009). Relativity theory and time perception: single or multiple clocks? PLoS ONE 4:6268. doi: 10.1371/journal.pone.0006268

Buhusi, C. V., and Meck, W. H. (2010). "Timing behavior," in Encyclopedia of Psychopharmacology, ed I. Stolerman (Berlin: Springer), 1319-1323.

Buhusi, C. V., and Oprisan, S. A. (2013). Time-scale invariance as an emergent property in a perceptron with realistic, noisy neurons. Behav. Process. 95, 60-70. doi: 10.1016/j.beproc.2013.02.015

Buhusi, C. V., Oprisan, S. A., and Buhusi, M. (2016). Clocks within clocks: timing by coincidence detection. Curr. Opin. Behav. Sci. 8, 207-213. doi: 10.1016/j.cobeha.2016.02.024

Buhusi, C. V., Paskalis, J.-P. G., and Cerutti, D. (2006). Time-sharing in pigeons: independent effects of gap duration, position and discriminability from the timed signal. Behav. Process. 71, 116-125. doi: 10.1016/j.beproc.2005.10.006

\section{AUTHOR CONTRIBUTIONS}

SO wrote the manuscript and derived the mathematical or computational results. $\mathrm{MB}$ and $\mathrm{CB}$ reviewed the manuscript.

\section{FUNDING}

The writing of this article was supported by NSF-IOS CAREER award 1054914 to SA, NIH grant NS090283 to MB, and NIH grant MH73057 to CB.

\section{ACKNOWLEDGMENTS}

SO acknowledges support from the National Center for Research Resources (5 P20 RR016461) and the National Institute of General Medical Sciences (8 P20 GM103499) from the National Institutes of Health, and a grant from the Howard Hughes Medical Institute to the College of Charleston as part of their 2012 Undergraduate Science Education Competition. SO acknowledges R\&D grant support from the College of Charleston.

Buhusi, M., Olsen, K., and Buhusi, C. V. (2017). Increased temporal discounting after chronic stress in chl1-deficient mice is reversed by 5 -ht $2 \mathrm{c}$ agonist ro 60-0175. Neuroscience 357, 110-118. doi: 10.1016/j.neuroscience.2017. 05.047

Canal, C., Stutz, S., and Gold, P. (2005). Glucose injections into the dorsal hippocampus or dorsolateral striatum of rats prior to t-maze training: modulation of learning rates and strategy selection. Learn. Mem. 12, 367-374. doi: 10.1101/lm.88205

Catania, A. C., and Reynolds, G. S. (1968). A quantitative analysis of the responding maintained by interval schedules of reinforcement. J. Exp. Anal. Behav. 11, 327-383. doi: 10.1901/jeab.1968.11-s327

Christakou, A., Robbins, T. W., and Everitt, B. J. (2004). Prefrontal cortical-ventral striatal interactions involved in affective modulation of attentional performance: Implications for corticostriatal circuit function. J. Neurosci. 24, 773-780. doi: 10.1523/JNEUROSCI.0949-03.2004

Church, R. M. (1984). Properties of the Internal Clock. New York, NY: New York Academy of Sciences.

Church, R. M. (2003). A Concise Introduction to Scalar Timing Theory. Boca Raton, FL: CRC Press. 3-22. doi: 10.1201/9780203009574.sec1

Church, R. M., and Broadbent, H. A. (1990). Alternative representations of time, number, and rate. Cognition 37, 55-81.

Church, R. M., and Broadbent, H. A. (1991). A Connectionist Model of Timing. Hillsdale, NJ: Lawrence Erlbaum Associates.

Church, R. M., Lacourse, D., and Crystal, J. (1998). Temporal search as a function of the variability of interfood intervals. J. Exp. Psychol. Anim. Behav. Process. 24, 291-315.

Church, R. M., Meck, W. H., and Gibbon, J. (1994). Application of scalar timing theory to individual trials. J. Exp. Psychol. Anim. Behav. Process. 20, 135-155.

Daniels, C. W., and Sanabria, F. (2017). Interval timing under a behavioral microscope: dissociating motivational and timing processes in fixed-interval performance. Learn. Behav. 45, 29-48. doi: 10.3758/s13420-016-0234-1

Datta, S., Siwek, D. F., Patterson, E. H., and Cipolloni, P. B. (1998). Localization of pontine pgo wave generation sites and their anatomical projections in the rat. Synapse 30, 409-423. doi: 10.1002/(SICI)1098-2396(199812)30:4<409::AIDSYN8>3.0.CO;2-\#

Egorov, A. V., Hamam, B. N., Fransen, E., Hasselmo, M. E., and Alonso, A. A. (2002). Graded persistent activity in entorhinal cortex neurons. Nature 420, 173-178. doi: 10.1038/nature01171

Gallistel, C. (2007). Flawed foundations of associationism? Comments on machado and silva. Am. Psychol. 62, 682-685. doi: 10.1037/0003-066X.62.7.682 
Gallistel, C., and Gibbon, J. (2000). Time, rate, and conditioning. Psychol. Rev. 107, 289-344. doi: 10.1037/0033-295X.107.2.289

Gallistel, C. R. (1990). The Organization of Behavior. Cambridge, MA: MIT Press.

Garvert, M. M., Dolan, R. J., and Behrens, T. E. (2017). A map of abstract relational knowledge in the human hippocampal? Entorhinal cortex. eLife 6:e17086. doi: 10.7554/eLife.17086

Gibbon, J. (1977). Scalar expectancy theory and weber's law in animal timing. Psychol. Rev. 84, 279-325. doi: 10.1037/0033-295X.84.3.279

Gibbon, J. (1991). Origins of scalar timing. Learn. Motiv. 22, 3-38. doi: 10.1016/0023-9690(91)90015-Z

Gibbon, J., and Church, R. M. (1984). Sources of Variance in an Information Processing Theory of Timing. Hillsdale, NJ: Erlbaum.

Gibbon, J., Church, R. M., Fairhurst, S., and Kaceinik, A. (1988). Scalar expectancy theory and choice between delayed rewards. Psychol. Rev. 95, 102-114.

Gibbon, J., Church, R. M., and Meck, W. H. (1984). Scalar timing in memory. Ann. N.Y. Acad. Sci. 423, 52-77.

Gibbon, J., Malapani, C., Dale, C. L., and Gallistel, C. R. (1997). Toward a neurobiology of temporal cognition: advances and challenges. Curr. Opin. Neurobiol. 7, 170-184.

Glenberg, A., Bradley, M. M., Stevenson, J. A., Kraus, T. A., Tkachuk, M. J., and Gretz, A. L. (1980). A two-process account of long-term serial position effects. J. Exp. Psychol. Hum. Learn. Mem. 6, 355-369. doi: 10.1037/0278-7393.6.4.355

Groenewegen, H. J., de Graaf, Y. G., and Smeets, W. J. (1999). Integration and segregation of limbic cortico-striatal loops at the thalamic level: an experimental tracing study in rats. J. Chem. Neuroanat. 16, 167-185. doi: 10.1016/S0891-0618(99)00009-5

Gu, B., Cheng, R., Yin, B., and Meck, W. H. (2011). Quinpirole-induced sensitization to noisy/sparse periodic input: temporal synchronization as a component of obsessive-compulsive disorder. Neuroscience 179, 143-150. doi: 10.1016/j.neuroscience.2011.01.048

Harrington, D. L., Haaland, K. Y., and Hermanowicz, N. (1998). Temporal processing in the basal ganglia. Neuropsychology 12, 3-12.

Houk, J. (1995). "Information processing in modular circuits linking basal ganglia and cerebral cortex," in Models of Information Processing in the Basal Ganglia, eds J. Houk, J. Davis, and D. Beiser (Cambridge: MIT Press), 3-10.

Houk, J., Adams, J., and Barto, A. (1995). A Model of How the Basal Ganglia Generate and Use Neural Signals That Predict Reinforcement. Cambridge, MA: MIT Press. 249-270

Howard, M. W., MacDonald, C. J., Tiganj, Z., Shankar, K. H., Du, Q., Hasselmo, M. E., et al. (2014). A unified mathematical framework for coding time, space, and sequences in the hippocampal region. J. Neurosci. 34, 4692-4707. doi: 10.1523/JNEUROSCI.5808-12.2014

Howard, M. W., Youker, T. E., and Venkatadass, V. (2008). The persistence of memory: contiguity effects across several minutes. Psychon. Bull. Rev. 15, 58-63. doi: 10.3758/PBR.15.1.58

Jin, D. Z., Fujii, N., and Graybiel, A. M. (2009). Neural representation of time in cortico-basal ganglia circuits. Proc. Natl. Acad. Sci. U.S.A. 106, 19156-19161. doi: 10.1073/pnas.0909881106

Jones, C., and Jahanshahi, M. (2011). Dopamine modulates striato-frontal functioning during temporal processing. Front. Integr. Neurosci. 5:70. doi: $10.3389 /$ fnint.2011.00070

Killeen, P. R., and Fetterman, J. G. (1988). A behavioral theory of timing. Psychol. Rev. 95, 274-295.

Killeen, P. R., and Fetterman, J. G. (1993). The behavioral theory of timing: transition analyses. J. Exp. Anal. Behav. 59, 411-422. doi: 10.1901/jeab.1993.59-411

Kraus, B. J., Robinson, R. J., White, J. A., Eichenbaum, H., and Hasselmo, M. E. (2013). Hippocampal "time cells": time versus path integration. Neuron 78, 1090-1101. doi: 10.1016/j.neuron.2013.04.015

Leak, T. M., and Gibbon, J. (1995). Simultaneous timing of multiple intervals: implications of the scalar property. J. Exp. Psychol. Anim. Behav. Process. 21, 3-19. doi: 10.1037/0097-7403.21.1.3

Lewis, P. A., and Miall, R. C. (2009). The precision of temporal judgement: milliseconds, many minutes, and beyond. Phil. Trans. R. Soc. Lond. B Biol. Sci. 364, 1897-1905. doi: 10.1098/rstb.2009.0020

Ludvig, E. A., Sutton, R. S., and Kehoe, E. J. (2008). Stimulus representation and the timing of reward-prediction errors in models of the dopamine system. Neural Comput. 20, 3034-3054. doi: 10.1162/neco.2008.1107-654
Luzardo, A., Alonso, E., and Mondragon, E. (2017). A Rescorla-Wagner driftdiffusion model of conditioning and timing. PLoS Comput. Biol. 13:e1005796. doi: 10.1371/journal.pcbi.1005796

Luzardo, A., Ludvig, E. A., and Rivest, F. (2013). An adaptive driftdiffusion model of interval timing dynamics. Behav. Process. 95, 90-99. doi: 10.1016/j.beproc.2013.02.003

MacDonald, C. J., Lepage, K. Q., Eden, U. T., and Eichenbaum, H. (2011). Hippocampal time cells bridge the gap in memory for discontiguous events. Neuron 71, 737-749. doi: 10.1016/j.neuron.2011.07.012

Machado, A. (1997). Learning the temporal dynamics of behavior. Psychol. Rev. $104,241-265$.

Machado, A., Malheiro, M. T., and Erlhagen, W. (2007). Learning to time: a perspective. J. Exp. Anal. Behav. 92, 423-458. doi: 10.1901/jeab.2009.92-423

Machado, A., and Silva, F. J. (2007). Toward a richer view of the scientific method: the role of conceptual analysis. Am. Psychol. 62, 671-681. doi: 10.1037/0003-066X.62.7.671

Malapani, C., and Fairhurst, S. (2002). Scalar timing in animals and humans. Learn. Motiv. 33, 156-176. doi: 10.1006/lmot.2001.1105

Mathis, A., Herz, A. V., and Stemmler, M. (2012). Optimal population codes for space: grid cells outperform place cells. Neural Comput. 24, 2280-2317. doi: 10.1162/NECO_a_00319

McKenna, J. T., and Vertes, R. P. (2004). Afferent projections to nucleus reuniens of the thalamus. J. Compar. Neurol. 480, 115-142. doi: 10.1002/cne.20342

O'Keefe, J. (1976). Place units in the hippocampus of the freely moving rat. Exp. Neurol. 51, 78-109. doi: 10.1016/0014-4886(76)90055-8

O'Keefe, J., and Burgess, N. (1996). Geometric determinants of the place fields of hippocampal neurons. Nature 381, 425-428. doi: 10.1038/381425a0

O'Keefe, J., and Recce, M. L. (1993). Phase relationship between hippocampal place units and the EEG theta rhythm. Hippocampus 3, 317-330. doi: 10.1002/hipo.450030307

Oprisan, S. A., Aft, T., Buhusi, M., and Buhusi, C. V. (2018). Scalar timing in memory: a temporal map in the hippocampus. J. Theor. Biol. 438, 133-142. doi: 10.1016/j.jtbi.2017.11.012

Oprisan, S. A., and Buhusi, C. V. (2011). Modeling pharmacological clock and memory patterns of interval timing in a striatal beat-frequency model with realistic, noisy neurons. Front. Integr. Neurosci. 5:52. doi: 10.3389/fnint.2011.00052

Oprisan, S. A., and Buhusi, C. V. (2013). How noise contributes to time-scale invariance of interval timing. Phys. Rev. E 87:052717. doi: 10.1103/PhysRevE.87.052717

Oprisan, S. A., and Buhusi, C. V. (2014). What is all the noise about in interval timing? Philos. Trans. R. Soc. Lond. B Biol. Sci. 369:20120459. doi: 10.1098/rstb.2012.0459

Packard, M., Cahill, L., and McGaugh, J. (1994). Amygdala modulation of hippocampal-dependent and caudate nucleus-dependent memory processes. Proc. Natl. Acad. Sci. U.S.A. 91, 8477-8481.

Parent, A. and Hazrati, L.-N. (1995a). Functional anatomy of the basal ganglia. I. The cortico-basal ganglia-thalamo-cortical loop. Brain Res. Rev. 20, 91-127. doi: 10.1016/0165-0173(94)00007-C

Parent, A., and Hazrati, L.-N. (1995b). Functional anatomy of the basal ganglia. II. The place of subthalamic nucleus and external pallidium in basal ganglia circuitry. Brain Res. Rev. 20, 128-154. doi: 10.1016/0165-0173(94) 00008-D

Pastalkova, E., Itskov, V., Amarasingham, A., and Buzsaki, G. (2008). Internally generated cell assembly sequences in the rat hippocampus. Science 321, 1322 1327. doi: 10.1126/science. 1159775

Penner, M., and Mizumori, S. (2012). Age-associated changes in the hippocampal-ventral striatum-ventral tegmental loop that impact learning, prediction, and context discrimination. Front. Aging Neurosci. 4:22. doi: 10.3389/fnagi.2012.00022

Perin, R., Berger, T. K., and Markram, H. (2011). A synaptic organizing principle for cortical neuronal groups. Proc. Natl. Acad. Sci. U.S.A. 108, 5419-5424 doi: 10.1073/pnas.1016051108

Rakitin, B. C., Gibbon, J., Penney, T. B., Malapani, C., Hinton, S. C., and Meck, W. H. (1998). Scalar expectancy theory and peak-interval timing in humans. J. Exp. Psychol. Anim. Behav. Process. 24, 15-33. doi: 10.1037/0097-7403.24.1.15

Schapiro, A. C., Turk-Browne, N. B., Norman, K. A., and Botvinick, M. M. (2015). Statistical learning of temporal community structure in the hippocampus. Hippocampus 26, 3-8. doi: 10.1002/hipo.22523 
Schiller, D., Eichenbaum, H., Buffalo, E. A., Davachi, L., Foster, D. J., Leutgeb, S., et al. (2015). Memory and space: towards an understanding of the cognitive map. J. Neurosci. 35, 13904-13911. doi: 10.1523/JNEUROSCI.261815.2015

Schultz, W. (2002). Getting formal with dopamine and reward. Neuron 36, 241263. doi: 10.1016/S0896-6273(02)00967-4

Silkis, I. (2008). A mechanism for influencing the septo-hippocampal theta rhythm by dopamine through the basal ganglia. Neurochem. J. 2, 157-163. doi: $10.1134 / \mathrm{S} 1819712408030045$

Simen, P., Balci, F., deSouza, L., Cohen, J., and Holmes, P. (2011a). Interval timing by long-range temporal integration. Front. Integr. Neurosci. 5:28. doi: $10.3389 /$ fnint. 2011.00028

Simen, P., Balci, F., deSouza, L., Cohen, J. D., and Holmes, P. (2011b). A model of interval timing by neural integration. J. Neurosci. 31, 9238-9253. doi: 10.1523/JNEUROSCI.3121-10.2011

Tam, S., and Bonardi, C. (2012a). Dorsal hippocampal involvement in appetitive trace conditioning and interval timing. Behav. Neurosci. 126, 258-269. doi: $10.1037 / \mathrm{a} 0027164$

Tam, S., and Bonardi, C. (2012b). Dorsal hippocampal lesions disrupt pavlovian delay conditioning and conditioned-response timing. Behav. Brain Res. 230, 259-267. doi: 10.1016/j.bbr.2012.02.016

Tam, S., Jennings, D., and Bonardi, C. (2013). Dorsal hippocampal involvement in conditioned-response timing and maintenance of temporal information in the absence of the CS. Exp. Brain Res. 227, 547-559. doi: 10.1007/s00221-013-3530-4

Tam, S., Jennings, D., and Bonardi, C. (2015). Effects of dorsal hippocampal damage on conditioning and conditioned-response timing: A pooled analysis. Hippocampus 25, 444-459. doi: 10.1002/hipo. 22381

Teki, S., Grube, M., and Griffiths, T. (2012). A unified model of time perception accounts for duration-based and beat-based timing mechanisms. Front. Integr. Neurosci. 5:90. doi: 10.3389/fnint.2011.00090
Thierry, A.-M., Gioanni, Y., Degenetais, E., and Glowinski, J. (2000). Hippocampo-prefrontal cortex pathway: anatomical and electrophysiological characteristics. Hippocampus 10, 411-419. doi: 10.1002/1098-1063(2000)10:4<411::AID-HIPO7>3.0.CO;2-A

Tiganj, Z., Hasselmo, M., and Howard, M. (2015). A simple biophysically plausible model for long time constants in single neurons. Hippocampus 25, 27-37. doi: 10.1002/hipo.22347

Vertes, R. P. (2001). Analysis of projections from the medial prefrontal cortex to the thalamus in the rat, with emphasis on nucleus reuniens. J. Compar. Neurol. 442, 163-187. doi: 10.1002/cne.10083

Voorn, P., Vanderschuren, L., Groenewegen, H., Robbins, T., and Pennartz, C. (2004). Putting a spin on the dorsal-ventral divide of the striatum. Trends Neurosci. 27, 468-474. doi: 10.1016/j.tins.2004.06.006

Wang, Y., Romani, S., Lustig, B., Leonardo, A., and Pastalkova, E. (2015). Theta sequences are essential for internally generated hippocampal firing fields. Nat. Neurosci. 18, 282-288. doi: 10.1038/nn.3904

Yin, B., and Meck, W. H. (2014). Comparison of interval timing behaviour in mice following dorsal or ventral hippocampal lesions with mice having d-opioid receptor gene deletion. Phil. Trans. R. Soc. B 369:20120466. doi: $10.1098 /$ rstb.2012.0466

Conflict of Interest Statement: The authors declare that the research was conducted in the absence of any commercial or financial relationships that could be construed as a potential conflict of interest.

Copyright (c) 2018 Oprisan, Buhusi and Buhusi. This is an open-access article distributed under the terms of the Creative Commons Attribution License (CC BY). The use, distribution or reproduction in other forums is permitted, provided the original author(s) and the copyright owner(s) are credited and that the original publication in this journal is cited, in accordance with accepted academic practice. No use, distribution or reproduction is permitted which does not comply with these terms. 